\title{
Public health response to outbreaks of Avian Influenza A(H5N2) and (H5N1) among poultry - British Columbia, December 2014-February 2015
}

\author{
Murti $\mathbf{M}^{1^{*}}$, Skowronski $\mathrm{D}^{2}$, Lem $\mathbf{M}^{1}$, Fung $\mathrm{C}^{1}$, Klar $\mathrm{S}^{1}$, Bigham $\mathbf{M}^{1}$, Loadman $\mathrm{S}^{1}$, Chambers $\mathrm{C}^{2}$, Pritchard $\mathrm{J}^{3}$, \\ Lee $\mathbf{V}^{1}$
}

${ }^{1}$ Fraser Health Authority, Surrey, BC
${ }_{2}$ BC Centre for Disease Control, Vancouver, BC
${ }^{3}$ Ministry of Agriculture, Abbotsford, BC

*Correspondence: Michelle.Murti@fraserhealth.ca

\begin{abstract}
In December 2014, the first detection in Canada of a highly pathogenic avian influenza A (HPAl) virus was reported in poultry within the Fraser Health Authority of British Columbia. It was the second outbreak of HPAI from Eurasian $\mathrm{H} 5$ reassortment viruses in North America. The Fraser Health Authority provided the lead public health coordination for this response as well as consultation and support to the occupational health response.

The public health response focused on contact tracing, monitoring and follow-up for household, farm worker and other community contacts exposed on the affected farms. A total of 50 contacts were identified. Contacts received daily active monitoring by public health nurses for seven days from their last exposure and were advised to selfmonitor until day 10. All contacts and other household members were recommended seasonal influenza vaccination to protect against further possible reassortment with human influenza viruses circulating within the community at the time. A total of $26(52 \%)$ contacts were recommended chemoprophylaxis for ongoing exposure to the affected barns and flocks, of whom only $11(42 \%)$ initiated this. During the seven-day active surveillance period, four contacts developed acute respiratory symptoms and influenza B was identified in one individual.

Local area health care providers and acute care facilities were alerted to the outbreak and public messaging was provided regarding the human health risks from avian influenza. Collaboration between health and agriculture at the local, regional, provincial and federal levels was key to a rapid response to this outbreak.
\end{abstract}

\section{Introduction}

In December 2014, the first detection in North America of a highly pathogenic avian influenza A (HPAl) virus with Eurasian $\mathrm{H} 5$ lineage genes was reported in poultry within the Fraser Health Authority (FHA) of British Columbia (BC), Canada. This was a highly pathogenic avian influenza H5N2 subtype resulting from mixing (i.e. genetic reassortment) between Eurasian lineage highly pathogenic avian influenza H5N8 and low pathogenic avian influenza virus of North American wild bird lineage

The initial detection was followed by further reports of domestic and wild or captive bird infections with Eurasian lineage highly pathogenic avian influenza H5N8, H5N2 and H5N1 reassortment viruses in the Pacific Northwest of the United States (US) in December 2014 and January 2015 and additional detection of the same novel highly pathogenic avian influenza H5N1 reassortment virus in poultry in the FHA in February $2015(1,2)$.

These detections represent the first outbreak of highly pathogenic avian influenza from Eurasian $\mathrm{H} 5$ reassortment viruses in North America. The term "highly pathogenic" refers to the spectrum of illness seen in birds, not humans. Affected flocks have experienced severe disease and high death rates, but there have been no human cases associated with these $\mathrm{H} 5$ viruses. In general, human infections with avian influenza viruses are rare and do not spread easily from person to person; however surveillance for animal-human transmission is essential for monitoring for the risk of human illness, particularly with a novel reassortment virus. This report provides details related to the public health response to the highly pathogenic avian influenza H5N2 and H5N1 reassortment virus detections in poultry by the Fraser Health Authority. 
Avian influenza virus infection in birds

Between December 1 and 19, 2014, the FHA, located within the south western Lower Mainland Region of BC which borders on the US, was notified of 11 commercial and one non-commercial poultry premises infected with highly pathogenic avian influenza H5N2. The report was the first regarding the Eurasian lineage highly pathogenic avian influenza H5 virus in North America. It is the second outbreak of an HPAI H5N2 strain in North America, after an outbreak in Pennsylvania in 1983, and previous outbreaks due to LPAI H5N2 viruses of North American lineage had been reported in West Virginia in 2007, British Columbia in 2009, and Manitoba in 2010 (3). Phylogenetic analysis of the HPAI H5N2 virus associated with the 2014 outbreak reveals that it is a novel reassortant virus A/turkey/BC/FAV10/2014 (H5N2) containing gene segments (GenBank Accession Numbers: KP307954-KP307961) from a Eurasian lineage HPAI H5N8 virus (including the Eurasian H5 clade 2.3.4.4 gene) and segments (including the N2 gene) from a typical North American LPAI virus of wild bird origin $(4,5)$.

Subsequent to the HPAI H5N2 outbreak in BC, the United States Department of Agriculture (USDA) reported several detections of novel reassortant HPAl viruses of Eurasian H5 lineage (H5N2, H5N8, and H5N1) in domestic and wild or captive birds in Oregon, Washington, Idaho, Utah and California between December 2014 and February 2015 (6). These were the first detections of HPAl subtypes H5N8 and H5N1 in birds in North America and are believed to have been introduced through co-mingling of wild birds. On February 6, 2015, the FHA was also notified of a non-commercial egg layer flock farm where HPAI A H5N1 was identified (A/Chicken/BC/FAV2/2015 (H5N1)), with sequencing identifying the same North American wild bird lineage and nearly identical H5N1 virus as had been recently detected in Washington State (1). This strain of H5N1 is genetically different from the avian $\mathrm{H} 5 \mathrm{~N} 1$ viruses that had caused human illness in other countries.

In total, the 11 farms and two backyard flocks affected within the region included a mix of turkey $(n=3)$, broiler breeder $(n=7)$, table egg layer $(n=1)$ and non-commercial flocks $(n=2$, including one with H5N1 reassortment). Over 245,000 birds have either died from the disease or have been humanely destroyed as part of the biosecurity response to the HPAI H5N2 and H5N1 detections in poultry in the region, coordinated through the Canadian Food Inspection Agency (CFIA), the BC Ministry of Agriculture and partner agencies (7).

\section{Public health response measures}

Building on previous avian influenza outbreaks in the region, notably the experience during the $2004 \mathrm{H} 7 \mathrm{~N} 3$ outbreak, the FHA's regional public health response has focused on contact tracing, monitoring and follow-up for household, farm worker and other community contacts exposed on the affected farms $(8,9)$. The FHA does not conduct contact tracing for CFIA contractors and other individuals occupationally exposed as part of the biosecurity response.

A total of 50 contacts were identified from the 13 affected premises: $35(70 \%)$ were male and the median age was 42 years old (range: 12-75 years). Contacts were assessed for their ongoing exposure and last date of exposure to the affected birds and were educated on the signs and symptoms of influenza-like illness (ILI) and instructed on self-monitoring and immediate reporting if any ILI symptoms developed. Contacts received daily active monitoring by public health nurses for seven days from their last exposure and advised to self-monitor until day 10. Given the ongoing destruction and disposal measures on affected premises, contacts also received a follow-up phone call at day 17 to confirm no new exposures and to ensure that they remained asymptomatic.

All contacts and other household members were recommended seasonal influenza vaccination to protect against further possible reassortment with human influenza viruses circulating within the community at that time, notably $A(H 3 N 2)(10)$. A total of $26(52 \%)$ were immunized either from previously in the season, or at the time of follow-up by public health. Contacts with ongoing exposure to the affected barns and flocks at the time of initial follow-up were recommended to initiate with antiviral chemoprophylaxis with oseltamivir and continue with daily dosing until seven days from last exposure. A total of $26(52 \%)$ were recommended chemoprophylaxis, of whom only 11 $(42 \%)$ initiated the antiviral prophylaxis.

During the seven-day active surveillance period, four contacts developed acute respiratory symptoms of cough or runny nose, but did not report fever and so did not meet the case definition for ILI. Nasopharyngeal swabs were obtained from all four symptomatic contacts. Influenza B was identified in one individual and the other three specimens were negative for influenza A and B. Two of the symptomatic contacts initiated treatment doses of oseltamivir with the onset of symptoms as a precautionary measure while nasopharyngeal swab results were pending. 
Local area health care providers and acute care facilities were alerted to the outbreak in early December and provided recommendations on the evaluation and management of symptomatic patients associated with infected farms. The FHA also disseminated public messaging on the human health risks from avian influenza to area poultry and swine farms through industry association liaisons. Messages included recommendations on receiving the seasonal influenza vaccine (publicly funded for all poultry workers in $\mathrm{BC}$ ) and the routine use of personal protective equipment. CFIA is the lead agency for coordination of the occupational health and safety for those employed as part of the biosecurity response.

Collaboration between health and agriculture at the local, regional, provincial and federal levels enabled a rapid response to this outbreak and the FHA has led public health coordination including consultation and support to the occupational health response.

\section{Conclusion}

The threat of pathogenic avian influenza from Asia (e.g., H5N1 and H7N9) with pandemic potential for humans requires vigilance in identifying changing epidemiology and novel reassortments. The current outbreak of HPAI H5N8 in poultry in Europe is the first European detection of this strain, previously detected in wild birds in Asia (11). The identification of a Eurasian lineage HPAI H5 virus in North America poses a concern for the potential introduction of the pathogenic zoonotic avian influenza strain from Asia to North America. With H5N2 and H5N1 detections in $\mathrm{BC}$ and $\mathrm{H} 5 \mathrm{~N} 2, \mathrm{H} 5 \mathrm{~N} 8$, and H5N1 detections in five north western states in the US, there is an increased likelihood of human exposure and infection in Canada (1).

As of the end of February 2015, there have been no human cases of H5N2, H5N8 or H5N1 identified through the active surveillance associated with the recent outbreaks in North America. However, some avian influenza H5 viruses can cause human infection, as demonstrated by the widespread $\mathrm{A}(\mathrm{H} 5 \mathrm{~N} 1)$ outbreak in Asia and parts of Africa (718 cases/413 deaths from January 2003 to 23 January 2015) (12).

On a global level, almost all cases of human infection with avian influenza H5 viruses have been reported in persons in close contact with infected or dead birds. Human-to-human transmission is rare and the risk of community-level spread is low. Global preventive measures therefore focus on those in closest contact with affected birds. Updated guidance from the Centers for Disease Control and Prevention (CDC) has been issued for human exposures to these strain types (13). Ongoing bird surveillance (both farm and wild) for notifiable avian influenza continues within the affected region including timely public health follow-up for all individuals potentially exposed to infected birds.

\section{Acknowledgements}

The co-authors acknowledge the public health staff who contributed to the contact management, as well as local, provincial, national and international partners involved in this response, particularly Dr. John Pasick from the Canadian Food Inspection Agency. They would also like to acknowledge the farmers and others impacted by this outbreak.

\section{Conflict of interest}

None

\section{Funding}

None

\section{References}

(1) Jhung MA, Nelson DI. Outbreaks of avian influenza A (H5N2), (H5N8), and (H5N1) among birds - United States, December 2014-January 2015. MMWR. 2015;64(04):111.

(2) Pan American Health Organization (PAHO), World Health Organization (WHO). Epidemiological Alert. Detection and outbreaks of avian influenza due to reassortant viruses, public health implications for the Americas. Washington: PAHO; February 6, 2015.

http://www.paho.org/hq/index.php?option=com_docman\&task=doc_view\&gid=29005+\&ltemid=999999\&lang=en. 
(3) World Animal Health Information Database (WAHID) Interface [Internet]. Home Page. Paris: World Organization for Anmal Health; December 30, 2013.

http://www.oie.int/wahis_2/public/wahid.php/Wahidhome/Home.

(4) Pasick J, Berhane Y, Joseph T, Bowes V, Hisanaga T, Handel K, Alexandersen S. Reassortant highly pathogenic influenza. $\mathrm{A}(\mathrm{H} 5 \mathrm{~N} 2)$ virus containing Eurasian lineage $\mathrm{H} 5$ and four other gene segments from Eurasian H5N8 in Canada, 2014. GenBank sequences deposited by Canadian Food Inspection Agency. Accession Numbers KP307954-KP307961.

(5) Ip HS, Torchetti MK, Crespo R, Kohrs P, DeBruyn P, Mansfield KG, et al. Novel Eurasian highly pathogenic influenza A H5 viruses in wild birds, Washington, USA, 2014. Emerg Infect Dis. 2015 May . http://dx.doi.org/10.3201/eid2105.142020

(6) USDA [Internet]. Update on avian influenza findings in the Pacific Flyway. Washington: USDA; February $24,2015$. http://www.aphis.usda.gov/wps/portal/?urile=wcm:path:/aphis_content_library/sa_our_focus/sa_animal_health/sa_ani mal_disease_information/sa_avian_health.

(7) Canadian Food Inspection Agency. Avian influenza - British Columbia infected premises. Ottawa: CFIA; February 23, 2015. http://www.inspection.gc.ca/animals/terrestrial-animals/diseases/reportable/ai/2014-2015-ai-investigation-inbc/infected-premises/eng/1418340527324/1418340584180.

(8) Skowronski DM, Li Y, Tweed A, Tam TWS, Petric M, David ST, Larder A, Birch P, Marra F, Seto J, Bastien N, King A, Lee SW, Prendergast $P$, Krajden M, Brunham R. Protective measures and human antibody response during an avian influenza H7N3 outbreak in poultry in British Columbia, Canada. CMAJ. 2007;176:47-53.

(9) Tweed SA, Skowronski DM, David ST, Larder A, Petric M, Lees M, et al. Human illness from avian influenza H7N3, British Columbia. Emerg Infect Dis. 2004;10:2196-9.

(10) BC Centre for Disease Control. Influenza surveillance reports. Vancouver: BCDC; 2015. http://www.bccdc.ca/dis-cond/DiseaseStatsReports/influSurveillanceReports.htm.

(11) European Centre for Disease Prevention and Control (ECDC). Outbreaks of highly pathogenic avian influenza A(H5N8) in Europe. Solna Sweden: ECDC; November 20, 2014. http://www.ecdc.europa.eu/en/publications/Publications/H5N8-influenza-Europe-rapid-risk-assessment-20-November2014.pdf

(12) World Health Organization (WHO). Influenza at the human-animal interface. Summary and assessment as of 26 January 2015. Geneva: WHO; 2015.

http://www.who.int/influenza/human_animal_interface/Influenza_Summary_IRA_HA_interface_26January2015.pdf.pd f?ua $=1$.

(13) Centers for Disease Control and Prevention (CDC) [Internet]. Interim guidance on testing, specimen collection, and processing for patients with suspected infection with novel influenza $A$ viruses with the potential to cause severe disease in humans. Atlanta: CDC.

http://www.cdc.gov/flu/avianflu/severe-potential.html. 